A New Formulation of the Nonmetric STRAIN Problem in Multidimensional Scaling

Michael W. Trosset

Angust, 1993

(Revised April 1997)

TR93-30 



\title{
A New Formulation of the Nonmetric Strain Problem in Multidimensional Scaling
}

\author{
Michael W. Trosset*
}

April 1, 1997

\begin{abstract}
A natural extension of classical metric multidimensional scaling is proposed. The result is a new formulation of nonmetric multidimensional scaling in which the strain criterion is minimized subject to order constraints on the disparity variables. Innovative features of the new formulation include: the parametrization of the $p$-dimensional distance matrices by the positive semidefinite matrices of rank $\leq p$; optimization of the (squared) disparity variables rather than the configuration coordinate variables; and a new nondegeneracy constraint, which restricts the set of (squared) disparities rather than the set of distances. Solutions are obtained by means of an easily implemented gradient projection method for numerical optimization. The method is applied to two published data sets.
\end{abstract}

Key words: Classical multidimensional scaling, strain criterion, distance geometry, order constraints, nondegeneracy constraints, gradient projection.

*Adjunct Associate Professor, Department of Computational \& Applied Mathematics, Rice University, Houston, TX (email: trosset@caam.rice.edu). 


\section{Introduction}

The phrase multidimensional scaling (MDS) has different connotations for different researchers. Some interpret it very broadly, others quite narrowly. Carroll and Arabie (1980) have proposed a very broad taxonomy of MDS; however, the focus of the present paper is more narrow, in the tradition of Kruskal (1977), Kruskal and Wish (1978), and de Leeuw and Heiser (1982). The latter construed scaling to be techniques for constructing a configuration of points in a target metric space from information about interpoint distances and subsequently defined MDS to be scaling in the case that the target space is Euclidean; see Arabie (1991) for a review of scaling in the case of non-Euclidean target spaces.

The present paper makes very specific assumptions about the nature of the information provided about the interpoint distances. Formally, we define a symmetric $n \times n$ matrix $\Delta=\left(\delta_{i j}\right)$ to be a dissimilarity matrix if $\delta_{i j} \geq 0$ (nonnegative elements) and $\delta_{i i}=0$ (zero diagonal elements). This is a fairly narrow definition of dissimilarity. We adopt it in order to make use of Theorem 1, which is the basis for the results that we report. Henceforth, we assume that the data form a dissimilarity matrix. Notice that this assumption is very slightly weaker than assuming that the data satisfy a semimetric.

From a given dissimilarity matrix $\boldsymbol{\Delta}^{0}$, a two-way MDS algorithm constructs a configuration of points in a Euclidean space of specified dimensionality $p$. For a configuration $\mathbf{x}_{1}, \ldots, \mathbf{x}_{n} \in R^{p}$, the $n \times p$ configuration matrix $\mathbf{X}$ is the matrix whose rows are the $\mathbf{x}_{i}^{\prime}, i=1, \ldots, n$. From $\mathbf{X}$ it is easy to compute the Euclidean interpoint distance matrix $\mathbf{D}(\mathbf{X})=\left(d_{i j}\right)$. The objective of two-way metric MDS, as defined in this paper, is to construct a configuration for which the interpoint distances $d_{i j}$ closely approximate the given dissimilarities $\delta_{i j}^{0}$.

Again following de Leeuw and Heiser (1982), we observe that two-way MDS is concerned with the case of a single dissimilarity matrix, whereas three-way or individual differences MDS is concerned with the case that the data comprise several dissimilarity matrices. The present paper is exclusively concerned with twoway MDS, although we will have occasion to mention certain procedures that were developed for three-way MDS.

The description of MDS presented above does not specify precisely what it means to "approximate" dissimilarities with distances. In fact, different specifications define different MDS techniques. Both de Leeuw and Heiser (1982) and Trosset (1996) organized surveys of MDS along these lines. The latter survey, which describes computational aspects of two-way MDS from the perspective of numerical optimization, is particularly germane to the present paper.

Historically, MDS techniques that seek a configuration that minimizes some measure of discrepancy between the configuration's interpoint distances and the given dissimilarities $\delta_{i j}^{0}$ have been termed metric. In contrast, nonmetric techniques minimize some measure of discrepancy between the interpoint distances and a set of dissimilarity matrices whose elements $\delta_{i j}$ have the same (weak) rank ordering as the given $\delta_{i j}^{0}$. The present paper is concerned with nonmetric MDS. A consequence of this concern is that only the ordinal information in $\boldsymbol{\Delta}^{0}$ is used in any essential way, so that the techniques developed herein are also applicable to ordinal dissimilarity data.

To distinguish between the observed dissimilarity matrix $\Delta^{0}$ and the set of dissimilarity matrices whose elements $\delta_{i j}$ have the same ordering, some terminology is required. It is traditional to refer to the fixed $\delta_{i j}^{0}$ as the dissimilarities. Once again following de Leeuw and Heiser (1982), we will refer to the varying $\delta_{i j}$ as the disparities. This terminology is potentially confusing, as certain other authors, e.g. Takane, Young, and de Leeuw (1977), have used it differently. However, our use of the term will be consistent throughout this report. Furthermore, we will simply refer to the $\left(\delta_{i j}\right)^{2}$ as the squared disparities.

Most MDS techniques, metric or nonmetric, employ one of three popular measures of discrepancy. The stress criterion, proposed by Kruskal (1964a) for nonmetric MDS, is based on the squared errors between the distances and the disparities. The sstress criterion, popularized by Takane, Young, and de Leeuw (1977) for nonmetric MDS, is based on the squared errors between the squared distances and the squared disparities. Both stress and sstress are easily adapted for use in metric MDS. In contrast, the strain criterion, which is implicit in Torgerson's (1952) classic work on metric MDS, and which enjoys compelling computational advantages over stress and sstress, has not been adapted satisfactorily for use in nonmetric MDS. Thus, the purpose of the present paper is to consider a promising new formulation of the nonmetric strain problem for two-way MDS. 


\section{The Strain Criterion}

Metric MDS was pioneered by Richardson (1938) and Torgerson (1952), who based their work on a beautiful theorem from distance geometry, first proved by Schoenberg (1935) and subsequently rediscovered by Young and Householder (1938). To state (one version of) this result, let $\boldsymbol{\Delta}$ be a fixed dissimilarity matrix. Let $\boldsymbol{\Delta} * \boldsymbol{\Delta}$ denote the Hadamard product of $\Delta$ with itself, so that the elements of $\Delta * \Delta$ are the squared dissimilarities. Let $\tau: \mathbf{A} \rightarrow \mathbf{B}$ denote the linear double centering operator defined by

$$
b_{i j}=-\frac{1}{2}\left(a_{i j}-\bar{a}_{i .}-\bar{a}_{. j}+\bar{a}_{. .}\right)
$$

where we adopt the usual convention of averaging over the dotted subscripts. Then,

Theorem 1 A configuration with interpoint distances equal to specified dissimilarities $\Delta$ exists if and only if $\tau(\boldsymbol{\Delta} * \boldsymbol{\Delta})$ is a symmetric positive semidefinite matrix of rank $\leq p$. If the $n \times p$ configuration matrix $\mathbf{X}$ satisfies $\tau(\Delta * \Delta)=\mathbf{X} \mathbf{X}^{\prime}$, then $\mathbf{D}(\mathbf{X})=\Delta$.

Because of the relation $\tau(\mathbf{D}(\mathbf{X}) * \mathbf{D}(\mathbf{X}))=\mathbf{X} \mathbf{X}^{\prime}$, some authors refer to $\tau(\Delta * \Delta)$ as the matrix of approximate scalar products.

Theorem 1 suggests the possibility of comparing a distance matrix $\mathbf{D}$ and a dissimilarity matrix $\boldsymbol{\Delta}$ by comparing the matrices $\tau(\mathbf{D} * \mathbf{D})$ and $\tau(\boldsymbol{\Delta} * \boldsymbol{\Delta})$. This formulation of metric MDS is defined by the optimization problem

$$
\text { minimize }\|\tau(\mathbf{D} * \mathbf{D})-\tau(\boldsymbol{\Delta} * \boldsymbol{\Delta})\|_{F}^{2}, \quad \text { subject to } \quad \mathbf{D} \in D_{n}(p),
$$

where $\|\cdot\|_{F}$ denotes the Frobenius norm, i.e., the $L^{2}$ norm on $R^{n \times n}$, and $D_{n}(p)$ denotes the set of all $n \times n$ matrices whose elements can be realized as the interpoint distances of $n$ points in $R^{p}$. Problem (1) is implicit in Torgerson (1952) and explicit in Gower (1966) and Mardia (1978). The objective function in Problem (1) is the strain criterion, so dubbed by Carroll and Chang (1970a).

By Theorem 1, Problem (1) is equivalent to the following optimization problem:

$$
\text { minimize }\|\mathbf{B}-\tau(\boldsymbol{\Delta} * \boldsymbol{\Delta})\|_{F}^{2}, \quad \text { subject to } \mathbf{B} \in \Omega_{n}(p),
$$

where $\Omega_{n}(p)$ is the set of symmetric positive semidefinite $n \times n$ matrices of rank $\leq p$. Thus, the metric strain problem reduces to the problem of finding a symmetric positive semidefinite matrix of rank $\leq p$ that is nearest a specified symmetric matrix.

Formulated and popularized in the 1950s, the metric strain problem is from an era in which numerical computation was extremely expensive. Undoubtedly, one of the most compelling reasons for the classical formulation of MDS is the fact that the global solutions of Problem (2) can be explicitly characterized using the spectral decomposition of $\tau(\boldsymbol{\Delta} * \boldsymbol{\Delta})$. The following well-known results appear as Theorem 14.4.2 in Mardia, Kent, and Bibby (1979) and are a special case of Theorem 2 in Trosset (1997).

Theorem 2 Let $\lambda_{1} \geq \cdots \geq \lambda_{n}$ denote the ordered eigenvalues of $\tau\left(\boldsymbol{\Delta}_{*} * \boldsymbol{\Delta}\right)$. Let $\bar{\lambda}_{j}=\max \left(\lambda_{j}, 0\right)$ if $j=1, \ldots, p$ and $\bar{\lambda}_{j}=0$ if $j=p+1, \ldots, n$. Let $\mathbf{\Lambda}=\operatorname{diag}\left(\lambda_{1}, \ldots, \lambda_{n}\right)$ and $\overline{\mathbf{\Lambda}}=\operatorname{diag}\left(\bar{\lambda}_{1}, \ldots, \bar{\lambda}_{n}\right)$ be diagonal matrices. If the orthogonal matrix $\mathbf{U}$ satisfies $\tau(\boldsymbol{\Delta} * \boldsymbol{\Delta})=\mathbf{U} \mathbf{\Lambda} \mathbf{U}^{\prime}$, then $\mathbf{B}^{*}=\mathbf{U} \overline{\mathbf{\Lambda}} \mathbf{U}^{\prime}$ is a global minimizer of Problem (2).

Corollary 1 Let $\mathbf{u}_{1}, \ldots, \mathbf{u}_{p}$ denote orthonormal vectors such that $\tau(\boldsymbol{\Delta} * \boldsymbol{\Delta}) \mathbf{u}_{j}=\lambda_{j} \mathbf{u}_{j}$. Then a configuration matrix $\mathbf{X}$ whose interpoint distances minimize the strain criterion is obtained by setting the columns of $\mathbf{X}$ equal to $\bar{\lambda}_{j}^{1 / 2} \mathbf{u}_{j}$ for $j=1, \ldots, p$.

Despite its computational advantages, the strain criterion fell from favor after Kruskal (1964a) proposed a rigorous basis for nonmetric two-way (one-mode) MDS using stress. In fact, it has turned out that the stress criterion does enjoy certain theoretical advantages over the strain criterion, at least for metric MDS. It follows from a theorem of de Leeuw (1984) that, except in very special cases, stress-optimal (unlike strain-optimal) configurations necessarily assign different locations to distinct objects. Meulman (1992) observed that strainoptimal (unlike stress-optimal) configurations necessarily underestimate actual interpoint distances, so that 
"when some distances are much more underestimated than others,... an inaccurate impression of similarity or indistinguishability might result." Our concern is not so much with the relative pros and cons of the strain criterion as it is with discovering precisely what can be accomplished if the strain criterion is used. In any event, detailed empirical investigations of the characteristics of configurations corresponding to different optimality criteria have not yet been attempted.

The strain criterion was revived by Carroll and Chang (1970b) when they introduced metric individual differences (three-way two-mode) MDS. In the 1970's, several attempts were made to formulate nonmetric MDS using strain. Unfortunately, it is difficult to assess precisely what was accomplished because most of this research was never published.

Motivated by an ad hoc device previously used in the TORSCA algorithm (Young and Torgerson 1967), Carroll and Chang (1970a, 1970b) also proposed NINDSCAL, a "quasi-nonmetric" algorithm for individual differences MDS. Although NINDSCAL is itself a three-way procedure, in the present two-way case it implies constructing the following iterative sequence, for which we use the superscripts $c$ and + to denote the current and subsequent iterates.

1. Compute the metric strain solution $\mathbf{X}^{c}$ from $\Delta^{c}$.

2. Compute the interpoint distances $\mathbf{D}\left(\mathbf{X}^{c}\right)$.

3. Perform an isotonic regression (Kruskal $1964 \mathrm{~b}$ ) of $\mathbf{D}\left(\mathbf{X}^{c}\right)$ on $\Delta^{0}$ to obtain $\Delta^{+}$.

It is entirely unclear just what this procedure accomplishes, as it alternates the minimization of two completely different objective functions. Furthermore, Carroll and Chang (1970b) reported that numerical experimentation had revealed that NINDSCAL "yields solutions in practice which are virtually indistinguishable from those attained by the 'metric' version."

Takane, Young, and de Leeuw (1977) subsequently introduced a method of nonmetric individual differences (three-way two-mode) MDS that uses sstress. For the special case of a single dissimilarity matrix (two-way one-mode MDS), this method is similar to Kruskal's (1964a) formulation of nonmetric MDS using stress. However, because Carroll and Chang (1970b) had already successfully proposed a method of metric individual differences MDS that uses strain, Takane, Young, and de Leeuw carefully considered the possibility of an analogous nonmetric approach and rejected two approaches to the nonmetric strain problem. The less interesting is formalized in the SCALAR algorithm of Young and Levinsohn (1974) that essentially applies Kruskal's nonmetric stress formulation to "the quasiscalar products matrix" $\tau\left(\Delta^{0} * \Delta^{0}\right)$. The difficulty with this approach is conceptual. The restriction to dissimilarity matrices $\Delta$ such that $\tau(\boldsymbol{\Delta} * \boldsymbol{\Delta})$ has the sarne elemental ordering as $\tau\left(\Delta^{0} * \Delta^{0}\right)$ has no apparent motivation. In the words of Takane, Young, and de Leeuw, "the measurement aspects of this approach are confusing."

Those authors also discussed the unpublished proposal by Carroll and Chang (1974) of a "fully nonmetric" strain procedure. In contrast to the SCALAR procedure, this procedure "assumes that the observed dissimilarities must be monotonic with a set of values from which the scalar products... are computed." We propose a procedure that also possesses this property. However, Takane, Young, and de Leeuw (1977) described Carroll's and Chang's optimization problem as "very complex," whereas the one presented here is quite simple and straightforward.

Finally, de Leeuw and Heiser (1982) described an algorithm called INDISCAL, developed by de Leeuw, Takane, and Young, which remains unpublished in English (personal communication from Y. Takane). INDISCA L expresses the strain criterion as a function of the configuration coordinate variables and the disparity variables; minimization is then accomplished by the method of alternating least squares. In contrast, we express the strain criterion as a function of positive semidefinite matrices and the squared disparity variables; minimization is then accomplished by a potentially more efficient optimization strategy that reduces the strain criterion to a function of just the squared disparity variables. Moreover, de Leeuw and Heiser state that "the major disadvantage of these procedures is that they may converge to non-global minima," whereas our formulation of the nonmetric strain problem seems to avoid this difficulty.

\section{A New Formulation}

Kruskal (1964a) suggested, as a rigorous basis for nonmetric MDS, replacing the fixed dissimilarity matrix $\Delta^{0}$ with the set of all dissimilarity matrices having the same rank ordering of their elements as $\Delta^{0}$. Specifically, 
suppose that the subdiagonal elements of $\boldsymbol{\Delta}^{0}$ are ordered as

$$
\delta_{i_{1} j_{1}}^{0} \leq \cdots \leq \delta_{i_{m} j_{m}}^{0}
$$

where $m=(n-1) n / 2$, and let $M\left(\Delta^{0}\right)$ denote the set of all dissimilarity matrices $\Delta$ for which

$$
\delta_{i_{1} j_{1}} \leq \cdots \leq \delta_{i_{m} j_{m}}
$$

Notice that $M\left(\boldsymbol{\Delta}^{0}\right)=M\left(\boldsymbol{\Delta}^{0} * \boldsymbol{\Delta}^{0}\right)$. Nonmetric MDS can then be defined as techniques for solving optimization problems of the form

$$
\text { minimize } \rho(\mathbf{D}, \boldsymbol{\Delta}), \quad \text { subject to } \quad \mathbf{D} \in D_{n}(p), \quad \boldsymbol{\Delta} \in M\left(\boldsymbol{\Delta}^{0}\right) .
$$

Of course, this is not the most general definition of nonmetric two-way MDS that is possible. For more general formulations, the interested reader is referred to the review articles cited in Section 1.

We would like to define the nonmetric strain problem by taking the objective function $\rho$ in Problem (4) to be the objective function in Problem (1), the metric strain problem. This direct approach fails because the sets $D_{n}(p)$ and $M\left(\Delta^{0}\right)$ always intersect at the origin of $R^{n \times n}$, so that a minimum value of strain equal to zero can always be obtained by simply placing all of the points in the configuration at the same location. Hence, to obtain a meaningful formulation of the nonmetric strain problem, we must also impose a nondegeneracy constraint to preclude such trivial solutions.

The traditional way of attempting to avoid degenerate solutions in nonmetric MDS with stress or sstress is through the use of a scaling factor in the objective function. As originally formulated by Kruskal (1964a), the nonmetric stress problem involves dividing the metric stress criterion by the squared Frobenius norm of the distances; as formulated by Takane Young, and de Leeuw (1977), the nonmetric sstress problem involves dividing the metric sstress criterion by the squared Froebenius norm of the squared disparities. Various alternative scaling factors were discussed by Kruskal and Carroll (1968).

As articulated by Kruskal and Carroll (1968), the introduction of a scaling factor was motivated by a perceived need "to provide invariance of the badness-of-fit function under changes of scale of the configuration." In contrast, from an optimization-theoretic perspective the scaling factor is a penalty function that enforces some constraint. Adopting the latter perspective, Trosset (1990) demonstrated that Kruskal's (1964a) original approach is equivalent to requiring that the Frobenius norm of the distances is no less than some positive constant. He also suggested that degenerate solutions might easily be avoided by requiring the disparities to be no less than the smallest positive dissimilarity. However, despite the computational simplicity that this nondegeneracy constraint affords, numerical experiments have revealed that it often leads to unacceptable solutions, at least when it is used in conjunction with the strain criterion. In the present paper, we will impose a new nondegeneracy constraint that requires the Frobenius norm of the disparities to be no less than the Frobenius norm of the dissimilarities.

The difficulty with the nondegeneracy constraint suggested by Trosset (1990) is that the magnitudes of the disparities are allowed to decrease too much in their attempt to become $p$-dimensional distances. Typically, many of them tend to their lower bound, so that practically no information from the dissimilarities is preserved. The present nondegeneracy constraint is a "conservation of total squared dissimilarity" restriction that precludes such behavior. Thus, our formulation of the nonmetric strain problem will be constructed to have the following intuitive interpretation: among all disparity matrices that allocate the total squared dissimilarity in accordance with the ordering imposed by the dissimilarities, find one that is most nearly a $p$-dimensional distance matrix.

Let

$$
r_{0}^{2}=\left\|\Delta^{0}\right\|_{F}^{2}=\sum_{i, j=1}^{n}\left(\delta_{i j}^{0}\right)^{2} .
$$

Then the following is our (first) formulation of the nonmetric strain problem:

$$
\text { minimize }\|\tau(\mathbf{D} * \mathbf{D})-\tau(\boldsymbol{\Delta} * \boldsymbol{\Delta})\|_{F}^{2}, \quad \text { subject to } \quad \mathbf{D} \in D_{n}(p), \quad \boldsymbol{\Delta} \in M\left(\boldsymbol{\Delta}^{0}\right), \quad \sum_{i, j=1}^{n} \delta_{i j}^{2} \geq r_{0}^{2} .
$$

Because $M\left(\boldsymbol{\Delta}^{0}\right)=M\left(\boldsymbol{\Delta}^{0} * \boldsymbol{\Delta}^{0}\right)$, the statement of this problem can be simplified by reparametrizing it in the squared disparities. Doing so results in replacing $\Delta * \Delta$ in the objective function with $\Delta$ and changes 
the nondegeneracy constraint to

$$
\Delta \in N\left(\Delta^{0}\right)=\left\{\Delta: \sum_{i, j=1}^{n} \delta_{i j} \geq r_{0}^{2}\right\} .
$$

If we also exploit Theorem 1 and write $\mathbf{B}=\tau(\mathbf{D} * \mathbf{D})$, then we obtain

$$
\text { minimize } \quad\|\mathbf{B}-\tau(\boldsymbol{\Delta})\|_{F}^{2}, \quad \text { subject to } \quad \mathbf{B} \in \Omega_{n}(p), \quad \boldsymbol{\Delta} \in M\left(\boldsymbol{\Delta}^{0}\right) \cap N\left(\boldsymbol{\Delta}^{0}\right) .
$$

This is a special case of a family of optimization problems studied by Trosset (1997).

The purpose of the present paper is to analyze carefully the above formulation of the nonmetric strain problem. Section 4 establishes some important properties of the constraint sets. Section 5 describes a method for solving Problem (5). Section 6 explores the character of the solutions for two illustrative examples. The paper concludes with an assessment of what has been accomplished and a discussion of several issues as yet unresolved.

\section{The Constraints}

Problem (5) is a nonlinear programming problem with a convex objective function. More precisely, the problem requires minimizing the distance (in Frobenius norm) between the sets $\Omega_{n}(p)$ and

$$
T=\left\{\mathbf{T}=\boldsymbol{\tau}(\boldsymbol{\Delta}): \boldsymbol{\Delta} \in M\left(\boldsymbol{\Delta}^{0}\right) \cap N\left(\boldsymbol{\Delta}^{0}\right)\right\} .
$$

In this section we establish that the minimum distance between these sets is attained and discuss how to compute projections into the constraint set $M\left(\boldsymbol{\Delta}^{0}\right) \cap N\left(\boldsymbol{\Delta}^{0}\right)$.

It is well-known that the set $\Omega_{n}(p)$ is a closed cone and that $\Omega_{n}(p)$ is not convex except when $p=n$. The set $M\left(\Delta^{0}\right)$ is a closed and convex cone and the set $N\left(\Delta^{0}\right)$ is closed and convex. Hence, the intersection of these sets is a closed and convex subset of a cone. Since $T$ is the image of this intersection under a linear map, it is also a closed and convex subset of a cone. Notice that the subset of this cone that is not in $T$ is bounded.

We begin by demonstrating that the minimum distance between $\Omega_{n}(p)$ and $T$ is attained and hence that optimal solutions of Problem (5) exist. By the above remarks, these facts are immediate consequences of the following result.

Lemma 1 Let $\Sigma_{1}$ and $\Sigma_{2}$ be closed subsets of the unit sphere in Euclidean space. Let $r: \Sigma_{1} \rightarrow[0, \infty)$ and $s: \Sigma_{2} \rightarrow[0, \infty)$ be bounded functions. Let $K_{1}=\left\{\alpha \mathbf{u}: \alpha \geq r(\mathbf{u}), \mathbf{u} \in \Sigma_{1}\right\}$ and $K_{2}=\left\{\beta \mathbf{v}: \beta \geq s(\mathbf{v}), \mathbf{v} \in \Sigma_{2}\right\}$. Then the minimum distance between $K_{1}$ and $K_{2}$ is attained.

Proof: Let $d<\infty$ denote the distance between $K_{1}$ and $K_{2}$. If $\mathbf{u} \in \Sigma_{1} \cap \Sigma_{2}$, then $\alpha \mathbf{u} \in K_{1} \cap K_{2}$ for $\alpha \geq \max (r(\mathbf{u}), s(\mathbf{u}))$, in which case $d=0$ is attained. Therefore, suppose that $\Sigma_{1}$ and $\Sigma_{2}$ are disjoint, in which case it follows from the Cauchy-Schwartz inequality and the compactness of $\Sigma_{1}$ and $\Sigma_{2}$ that

$$
\sup \left\{(\mathbf{u}, \mathbf{v}): \mathbf{u} \in \Sigma_{1}, \mathbf{v} \in \Sigma_{2}\right\} \leq 1-\epsilon
$$

for some $\epsilon>0$.

Now suppose that

$$
\begin{aligned}
d^{2} & =\lim _{k \rightarrow \infty}\left\|\alpha_{k} \mathbf{u}_{k}-\beta_{k} \mathbf{v}_{k}\right\|^{2} \\
& =\lim _{k \rightarrow \infty} \alpha_{k}^{2}-2 \alpha_{k} \beta_{k}\left(\mathbf{u}_{k}, \mathbf{v}_{k}\right)+\beta_{k}^{2} \\
& =\lim _{k \rightarrow \infty}\left(\alpha_{k}^{2}-\beta_{k}\right)^{2}+2 \alpha_{k} \beta_{k}\left[1-\left(\mathbf{u}_{k}, \mathbf{v}_{k}\right)\right] \\
& \geq \lim _{k \rightarrow \infty}\left(\alpha_{k}^{2}-\beta_{k}\right)^{2}+2 \alpha_{k} \beta_{k} \epsilon .
\end{aligned}
$$

Then $\left\{\alpha_{k}\right\}$ and $\left\{\beta_{k}\right\}$, hence $\left\{\alpha_{k} \mathbf{u}_{k}\right\}$ and $\left\{\beta_{k} \mathbf{v}_{k}\right\}$ must be bounded, and there must exist subsequences $\alpha_{k^{\prime}} \mathbf{u}_{k^{\prime}} \rightarrow \alpha_{*} \mathbf{u}_{*} \in K_{1}$ and $\beta_{k^{\prime}} \mathbf{v}_{k^{\prime}} \rightarrow \beta_{*} \mathbf{v}_{*} \in K_{2}$ such that $\left\|\alpha_{*} \mathbf{u}_{*}-\beta_{*} \mathbf{v}_{*}\right\|=d$. 
We now turn to the question of how to compute projections into the constraint set $M\left(\Delta^{0}\right) \cap N\left(\Delta^{0}\right)$. These computations will play a crucial role in the method for solving Problem (5) that we describe in Section 5. Let us write the constraint $M\left(\boldsymbol{\Delta}^{0}\right) \cap N\left(\boldsymbol{\Delta}^{0}\right)$ as the intersection of the constraints

$$
0 \leq \delta_{i_{1} j_{1}}^{1 / 2} \leq \cdots \leq \delta_{i_{m} j_{m}}^{1 / 2}
$$

and

$$
\sum_{i, j=1}^{n} \delta_{i j}=\sum_{i, j=1}^{n}\left[\delta_{i j}^{1 / 2}\right]^{2} \geq r_{0}^{2} .
$$

Constraint (6) defines a closed and convex cone; constraint (7) restricts attention to elements of that cone of norm no less than $r_{0}$. The problem of projecting (in $L^{2}$ norm) into (6) is the much-studied problem of isotonic regression, for which a variety of finite algorithms exist. In our numerical experiments, we have used a very efficient implementation, due to Grotzinger and Witzgall (1984), of the well-known Pool-Adjacent-Violators Algorithm. This implementation is easily programmed and requires only $O(m)$ operations.

Given that we can use isotonic regression to project into (6), we can exploit the following result to compute projections into the intersection of (6) and (7).

Lemma 2 Let $\mathbf{y}$ be a point and $K$ a closed and convex cone in Euclidean space. Suppose that $\overline{\mathbf{x}}$ is the point in $K$ nearest $\mathbf{y}$. If $\|\overline{\mathbf{x}}\|<c$, then the point in $\{\mathbf{x} \in K:\|\mathbf{x}\| \geq c\}$ nearest $\mathbf{y}$ is $c \overline{\mathbf{x}} /\|\overline{\mathbf{x}}\|$.

Proof: Let $\mathbf{x}_{1}=c \overline{\mathbf{x}} /\|\overline{\mathbf{x}}\|$ and let $\mathbf{x}_{2}$ be any point in $\{\mathbf{x} \in K:\|\mathbf{x}\| \geq c\}$ nearest $\mathbf{y}$. By hypothesis, the constraint $\|\mathbf{x}\| \geq c$ is active, so $\left\|\mathbf{x}_{2}\right\|=c=\left\|\mathbf{x}_{1}\right\|$. It follows that

$$
0 \leq\left\|\mathbf{y}-\mathbf{x}_{1}\right\|^{2}-\left\|\mathbf{y}-\mathbf{x}_{2}\right\|^{2}=\|\mathbf{y}\|^{2}-2\left(\mathbf{y}, \mathbf{x}_{1}\right)+\|\mathbf{x}\|^{2}-\|\mathbf{y}\|^{2}+2\left(\mathbf{y}, \mathbf{x}_{2}\right)-\left\|\mathbf{x}_{2}\right\|^{2}=2\left(\mathbf{y}, \mathbf{x}_{2}-\mathbf{x}_{1}\right)
$$

and hence, for any $\alpha \geq 0$, that

$$
\begin{aligned}
\left\|\mathbf{y}-\alpha \mathbf{x}_{1}\right\|^{2}-\left\|\mathbf{y}-\alpha \mathbf{x}_{2}\right\|^{2} & =\|\mathbf{y}\|^{2}-2 \alpha\left(\mathbf{y}, \mathbf{x}_{1}\right)+\|\mathbf{x}\|^{2}-\|\mathbf{y}\|^{2}+2 \alpha\left(\mathbf{y}, \mathbf{x}_{2}\right)-\left\|\mathbf{x}_{2}\right\|^{2} \\
& =2 \alpha\left(\mathbf{y}, \mathbf{x}_{2}-\mathbf{x}_{1}\right) \geq 0
\end{aligned}
$$

Then, setting $\alpha=\|\overline{\mathbf{x}}\| / c$ gives

$$
\|\mathbf{y}-\overline{\mathbf{x}}\|^{2} \geq\left\|\mathbf{y}-\alpha \mathbf{x}_{2}\right\|^{2},
$$

which, by the uniqueness of projection into a convex set, is impossible unless $\alpha \mathbf{x}_{2}=\overline{\mathbf{x}}$, i.e., $\mathbf{x}_{2}=\mathbf{x}_{1}$.

To apply Lemma 2 to the present case of projecting $\delta \in R^{m}$ into the intersection of (6) and (7), let $\bar{\delta}$ denote the projection of $\delta$ into (6), determined by isotonic regression. If $r^{2}=\sum_{i j} \bar{\delta}_{i j} \geq r_{0}^{2}$, then $\bar{\delta}$ lies in the intersection of (6) and (7) and we are done. If $r^{2}<r_{0}^{2}$, then $r_{0}^{2} \bar{\delta} / r^{2}$ is the projection of $\delta$ into the intersection of $(6)$ and $(7)$.

\section{Optimization}

Problem (5) is a special case of a more general family of optimization problems studied by Trosset (1997). In this section, we adapt methods developed for this family of problems to the specific problem at hand.

A crucial characteristic of Problem (5) is that there is a natural distinction between the sets of variables $\mathbf{B} \in \Omega_{n}(p)$ and $\boldsymbol{\Delta} \in M\left(\boldsymbol{\Delta}^{0}\right) \cap N\left(\boldsymbol{\Delta}^{0}\right)$. First, there are no mixed constraints, i.e. constraints that involve both sets of variables. Second, if one fixes either set of variables, then one obtains a less complicated optimization subproblem in the other set of variables. Following Parks (1985), we will call such nonlinear programs reducible.

Historically, there have been two important techniques for exploiting the structure of reducible programs. One technique is variable alternation, in which one successively alternates solving the aforementioned subproblems. The other technique is variable reduction, in which one set of variables is parametrized using the other. Specifically, one defines a function from one set of variables to the other by assigning to each fixed value of the first set a solution to the subproblem in the second set. An elementary introduction to both techniques is included in Trosset (1996). Trosset (1997) applied both methods to the problem of minimizing 
the distance (in Frobenius norm) between certain closed convex sets of symmetric matrices and certain closed subsets of the symmetric positive semidefinite matrices. Problem (5) is an example of such a problem.

In this paper, we employ the variable reduction strategy favored by Trosset (1997). To do so, we require an explicit expression for the minimum value of the strain criterion for a fixed matrix $\Delta$ of squared disparities. Following Theorem 2, let $\lambda_{1} \geq \cdots \geq \lambda_{n}$ denote the ordered eigenvalues of $\tau(\Delta)$. Then it is easily calculated that the global minimum of the (metric) strain criterion is given by the variable projection functional

$$
F_{p} \circ \tau(\boldsymbol{\Delta})=\sum_{i=1}^{p}\left[\lambda_{i}-\max \left(\lambda_{i}, 0\right)\right]^{2}+\sum_{i=p+1}^{n} \lambda_{i}^{2}=\sum_{i=1}^{p} \phi\left(\lambda_{i}\right)+\sum_{i=p+1}^{n} \psi\left(\lambda_{i}\right),
$$

where $\phi$ and $\psi$ denote the continuously differentiable functions $\phi(t)=t^{2} I_{(-\infty, 0]}(t)$ and $\psi(t)=t^{2}$. Our strategy for solving Problem (5) will be to minimize (8) as $\Delta$ varies subject to the constraint that it lies in $M\left(\boldsymbol{\Delta}^{0}\right) \cap N\left(\boldsymbol{\Delta}^{0}\right)$.

Before proceeding, we note that variable reduction techniques have been used previously to construct algorithms for MDS, most notably by Kruskal (1964a, 1964b). Variable reduction was first applied to the additive constant problem by Saito (1978); de Leeuw and Heiser (1982) and Trosset, Baggerly, and Pearl (1996) subsequently suggested using the variable projection functional $F_{p} \circ \tau$. Trosset (1995) considered minimizing $F_{p}$ o $\tau$ subject to bound constraints, which has applications to distance matrix completion problems and certain problems in molecular conformation.

Identifying $\Delta$ with its subdiagonal elements $\delta$, the reduced formulation of Problem (5) can be written as

$$
\begin{array}{ll}
\operatorname{minimize} & F_{p} \circ \tau\left(\delta_{i_{1} j_{1}}, \ldots, \delta_{i_{m} j_{m}}\right) \\
\text { subject to } & 0 \leq \delta_{i_{1} j_{1}} \leq \cdots \leq \delta_{i_{m} j_{m}}, \quad \delta_{i_{1} j_{1}}+\cdots+\delta_{i_{m} j_{m}} \geq r_{0}^{2} / 2
\end{array}
$$

This is a nonlinear program with linear inequality constraints, which we note are equivalent to the constraints (6) and (7).

Given a matrix $\Delta$ of squared disparities, let $\mathbf{T}=\tau(\boldsymbol{\Delta})$ have eigenvalues $\lambda_{1}(\mathbf{T}) \geq \cdots \geq \lambda_{n}(\mathbf{T})$. Let $\mathbf{B}^{*}(\mathbf{T})$ denote a solution of Problem (1) for the corresponding disparities and let $\mathbf{S}=\mathbf{B}^{*}(\mathbf{T})-\mathbf{T}$. Trosset (1997) demonstrated that the objective function $F_{p} \circ \tau$ is continuously differentiable at $\boldsymbol{\Delta}$ unless $\lambda_{p}(\mathbf{T})=$ $\lambda_{p+1}(\mathbf{T}) \geq 0$ and differentiated it with respect to the subdiagonal elements of $\boldsymbol{\Delta}$, obtaining

$$
\frac{\partial}{\partial \delta_{i_{k} j_{k}}} F \circ \tau(\delta)=\frac{2}{n^{2}} \mathbf{S}_{++}-\frac{2}{n}\left(\mathbf{S}_{i_{k}+}+\mathbf{S}_{j_{k}+}\right)+2 \mathbf{S}_{i_{k} j_{k}}
$$

where we sum over indices denoted by + .

For solving more general problems of which Problem (9) is an example, Trosset (1997) suggested a gradient projection method. Because the results of Section 4 provide an efficient way of projecting into the feasible set of Problem (9), we proceed to implement that suggestion. It should be possible to develop more efficient, second-order methods for solving Problem (9); however, Kruskal (1977) has defended gradient methods for MDS on the grounds that psychometric data is usually not sufficiently precise to warrant identifying extremely accurate solutions.

Gradient projection methods were originally proposed for convex programming by Goldstein (1964) and by Levitin and Polyak (1966). Let $f$ be a smooth convex function defined on a closed convex set $C \subset R^{m}$ and let $P$ denote projection into $C$. A gradient projection algorithm for the problem

$$
\text { minimize } f(\mathbf{z}) \quad \text { subject to } \quad \mathbf{z} \in C,
$$

is defined by specifying the step lengths $\alpha_{k}$ in the iterative sequence

$$
\mathbf{z}_{k+1}=P\left[\mathbf{z}_{k}-\alpha_{k} \nabla f\left(\mathbf{z}_{k}\right)\right] .
$$

In some gradient projection algorithms, e.g. McCormick and Tapia (1972), the step length is determined by a line search, i.e. $\alpha_{k}$ is chosen to minimize the univariate function

$$
g(\alpha)=f\left(P\left[\mathbf{z}_{k}-\alpha_{k} \nabla f\left(\mathbf{z}_{k}\right)\right]\right)
$$


An explicit procedure for determining appropriate step lengths was introduced by Bertsekas (1976) and improved by Calamai and Moré (1987) in the case of linear constraints.

When the objective function is not convex, the theory of gradient projection methods guarantees only that accumulation points of the sequence of iterates are constrained stationary points. In practice, because the sequence of function values is decreasing, such points typically will be local minimizers. For Problem (9), it appears that these local minimizers are typically global minimizers. This phenomenon is difficult to investigate, but studies in progress of closely related problems in which $F_{p} \circ \tau$ is minimized subject to bound constraints with a known global minimum have revealed few (if any) local minimizers that are not global minimizers.

\section{Two Examples}

We illustrate the viability of our formulation of the nonmetric strain problem by solving Problem (9) for two published data sets. The numerical results reported were obtained using the gradient projection method described by Bertsekas (1976). In practice, it appears that the existence of dissimilarity matrices at which $F$ is nondifferentiable is not a cause for concern. All computations were performed on a personal computer with an Intel 486DX processor using S-PLUS for Windows.

One of the troublesome characteristics of actual dissimilarities is the inevitable presence of ties, rendering the ordering of the dissimilarities in (3) nonunique. If an application warrants sufficient attention, then the most satisfactory approach is to replace (3) with an appropriate partial ordering of the dissimilarities. All the methods discussed in this paper remain applicable, but one must use an isotonic regression algorithm that can accomodate partial orderings. In our judgment, the illustrative nature of our examples argued against such an expenditure of time and energy. Instead, we broke ties lexicographically: if $\delta_{i j}^{0}=\delta_{k l}^{0}$ and either $i<k$ or $i=k$ and $j<l$, then we required $\delta_{i j} \leq \delta_{k l}$.

Our first illustration uses Wish's (Wish, 1971; Wish, Deutsch, and Biener, 1972; Kruskal and Wish, 1978) similarity data on perceptions of nations. In Wish's experiment, each of 18 psychology students rated the similarity of $m=66$ pairs of $n=12$ nations on a scale of 1 ("very different") to 9 ("very similar"). The mean similarity ratings are reported in Figure 7 of Kruskal and Wish (1978). Figure 1 of the present paper reproduces Kruskal's and Wish's two-dimensional nonmetric solution, which used stress formula one in the well-known KYST program (Kruskal, Young, and Seery 1973).

Insert Figure 1 about here.

To obtain a corresponding solution of the nonmetric strain problem, dissimilarities were defined by subtracting the mean similarity ratings from 9 . The sum of the squares of these $m=66$ dissimilarites is $r_{0}^{2} / 2=1514.65$. Their strain is $F \circ \tau\left(\boldsymbol{\Delta}^{0} * \boldsymbol{\Delta}^{0}\right)=936.22$, which means that the distance (in Frobenius norm) of the double centered matrix $\tau\left(\Delta^{0} * \Delta^{0}\right)$ from $\Omega_{12}(2)$ is 30.60. Ten iterations of gradient projection reduced the strain to 654.04 , which means that the distance between the feasible set of doubly centered squared disparity matrices and $\Omega_{12}(2)$ is 25.57. Computation was terminated because the $L^{2}$ distance between the vectors of $m=66$ squared disparities on iterations 9 and 10 was 0.58 and the corresponding difference in strain values was 0.04 .

A configuration corresponding to the minimal strain is plotted in Figure 2. Except for scale factor and orientation (both arbitrary), this configuration is fairly though not entirely similar to the configuration plotted in Figure 1. To facilitate interpretation, Kruskal and Wish (1978) imposed interpretative orthogonal axes on their configuration corresponding to (Cold War) Political Alignment ("Pro-Communist" vs. "Pro-Western") and Economic Development ("Underdeveloped" vs. "Developed"). The latter dimension is strikingly apparent in Figure 2, whereas grouping by political ideology is more pronounced in Figure 1. In addition, the strain configuration highlights the separation of Brazil, China, and the United States. It should be emphasized, of course, that neither configuration is the "correct" one. Each is the result of certain arbitrary choices, and presumably each reveals useful information about the data from which they were both constructed. 
We now consider one of the most famous data sets in the early history of nonmetric MDS. Ekman (1954) conducted an experiment in which each of 31 students rated the similarity of $m=91$ pairs of $n=14$ color stimuli on a scale of 0 ("no similarity at all") to 4 ("identity"). The stimuli were obtained using $12 \mathrm{~m} \mu$ halfband filters that transmitted light of central wavelengths ranging from 434 to $674 \mathrm{~m} \mu$. The mean similarity ratings were scaled to range from 0 to 1 , treated as a correlation matrix, and subjected to factor analysis. Ekman concluded that no less than five factors, which he identified as violet, blue, green, yellow, and red, were required to adequately account for variation in the empirical similarity measures.

In contrast, Shepard (1962) observed that, "Since variations in hue are usually represented by the familiar 'color circle' in two-dimensional space, Ekman's finding that five dimensions are required is rather surprising." He subjected the same similarity matrix to his own early version of nonmetric MDS and found that $p=2$ dimensions sufficed to account for most of the variation. The configuration of $n=14$ points constructed by MDS in $R^{2}$ was almost perfectly circular, and a clockwise ordering of the points placed the colors in order of increasing wavelength.

To obtain a corresponding solution using nonmetric strain, dissimilarities were defined by scaling the mean similarity ratings to range from 0 to 10 , then subtracting them from 10 . The sum of the squares of these $m=91$ dissimilarites is $r_{0}^{2} / 2=6133.10$. Their strain is $F \circ \tau\left(\Delta^{0} * \Delta^{0}\right)=3760.10$, so that the distance of $\tau\left(\Delta^{0} * \Delta^{0}\right)$ from $\Omega_{14}(2)$ is 61.32 . Eighteen iterations of gradient projection reduced the strain to 518.90 , so that the distance between the feasible set of doubly centered squared disparity matrices and $\Omega_{14}(2)$ is 22.78 . Computation was terminated because the $L^{2}$ distance between the vectors of $m=91$ squared disparities on iterations 17 and 18 was 0.21 and the corresponding difference in strain values was 0.003 .

A configuration corresponding to the minimal strain is plotted in Figure 3. This configuration also recovers the notion of a color circle-in fact, except for scaling and orientation, it is almost identical to Shepard's configuration. Thus, the methods proposed in the present paper replicate what has long been regarded as a major triumph of nonmetric MDS.

Insert Figure 3 about here.

\section{Discussion}

A natural scheme for classifying MDS procedures can be based on three characteristics. First, the disparities are typically either restricted to the observed dissimilarities (metric MDS) or allowed to vary subject to order constraints (nonmetric MDS). Second, the objective (or loss) function is typically stress, sstress, or strain. Third, the number of dissimilarity matrices is either one (two-way MDS) or more (three-way, or individual differences, MDS). To date, the two cells in this $2 \times 3 \times 2$ crosstabulation corresponding to nonmetric strain have been studied much less than the other ten. Thus, the work presented in this paper is, first and foremost, of considerable historical interest, filling one of the neglected gaps in the MDS mosaic.

Our formulation of nonmetric MDS using the strain criterion is distinguished by several innovative features. One is our parametrization of the $p$-dimensional dist ance matrices by the positive semidefinite matrices of rank $\leq p$, rather than by the $p$-dimensional configurations themselves. A second is our optimization of the squared disparity variables, rather than the configuration coordinate variables. Perhaps the most interesting feature of our formulation is our use of a nontraditional nondegeneracy constraint, which restricts the set of squared disparities rather than the set of distances. The study of nondegeneracy constraints other than the usual penalty function approach that was introduced by Kruskal (1964a) is still very much in its infancy. We hope that the success of the present formulation will stimulate further research along the same lines.

Finally, there needs to be a comprehensive comparative study of the characteristics of the solutions obtained by applying different nonmetric MDS procedures to the same data sets. Whatever the outcome of such a study, it is bound to be beneficial. If different procedures tend to produce very similar configurations, as in our examples, then a responsible decision can be made to prefer whichever procedures are computationally 
more efficient. If different procedures sometimes produce very different configurations, then knowledge of the differences is obviously essential to the intelligent choice of a procedure.

\section{Acknowledgments}

This research was supported in part by grant DMS-9622749 from the National Science Foundation and by the W. M. Keck Center for Computational Biology. I have greatly benefitted from discussions with J. Douglas Carroll, Jan de Leeuw, Timothy Havel, Yoshio Takane, and Richard Tapia; and from comments made by three referees and the editor.

\section{References}

Arabie, P. (1991). Was Euclid an unnecessarily sophisticated psychologist? Psychometrika, 56:567-587.

Bertsekas, D. P. (1976). On the Goldstein-Levitin-Polyak gradient projection method. IEEE Transactions on Automatic Control, 21:174-184.

Calamai, P. H. and Moré, J. J. (1987). Projected gradient methods for linearly constrained problems. Mathematical Programming, 39:93-116.

Carroll, J. D. and Arabie, P. (1980). Multidimensional scaling. Annual Review of Psychology, 31:607-649.

Carroll, J. D. and Chang, J.-J. (1970a). Analysis of individual differences in multidimensional scaling via an n-way generalization of "Eckart-Young" decomposition. Psychometrika, 35:283-319.

Carroll, J. D. and Chang, J.-J. (1970b). A "quasi-metric" version of INDSCAL, a procedure for individual differences multidimensional scaling. Paper presented at the Spring meeting of the Psychometric Society, Stanford, CA, March, 1970.

Carroll, J. D. and Chang, J.-J. (1974). Some methodological advances in INDSCAL. Paper presented at the Spring meeting of the Psychometric Society, Stanford, CA, April, 1974.

Critchley, F. (1988). On certain linear mappings between inner-product and squared-distance matrices. Linear Algebra and Its Applications, 105:91-107.

de Leeuw, J. (1984). Differentiability of Kruskal's Stress at a local minimum. Psychometrika, 49:111-113.

de Leeuw, J. and Heiser, W. (1982). Theory of multidimensional scaling. In Krishnaiah, P. R. and Kanal, I. N., editors, Handbook of Statistics, volume 2, chapter 13, pages 285-316. North-Holland Publishing Company, Amsterdam.

Ekman, G. (1954). Dimensions of color vision. Journal of Psychology, 38:467-474.

Goldstein, A. A. (1964). Convex programming in Hilbert space. Bulletin of the American Mathematical Society, 70:709-710.

Gower, J. C. (1966). Some distance properties of latent root and vector methods in multivariate analysis. Biometrika, 53:315-328.

Grotzinger, S. J. and Witzgall, C. (1984). Projections onto order simplexes. Applied Mathematics and Optimization, 12:247-270.

Kruskal, J. B. (1964a). Multidimensional scaling by optimizing goodness of fit to a nonmetric hypothesis. Psychometrika, 29:1-27.

Kruskal, J. B. (1964b). Nonmetric multidimensional scaling: A numerical method. Psychometrika, 29:28-42. 
Kruskal, J. B. (1977). Multidimensional scaling and other methods for discovering structure. In Enslein, K., Ralston, A., and Wilf, H. S., editors, Statistical Methods for Digital Computers, chapter 12, pages 296-339. John Wiley \& Sons, New York. Volume III of Mathematical Methods for Digital Computers.

Kruskal, J. B. and Carroll, J. D. (1968). Geometrical models and badness-of-fit functions. In Krishnaiah, P. R., editor, Multivariate Analysis, volume 2, pages 639-671. Academic Press, New York.

Kruskal, J. B. and Wish, M. (1978). Multidimensional Scaling. Sage Publications, Beverly Hills. Sage University Paper series on Quantitative Applications in the Social Sciences, 07-001.

Kruskal, J. B., Young, F. W., and Seery, J. B. (1973). How to use KYST, a very flexible program to do multidimensional scaling and unfolding. Technical report, AT\&T Bell Laboratories, Murray Hill, NJ.

Levitin, E. S. and Polyak, B. T. (1966). Constrained minimization problems. USSR Computational Mathematics and Mathematical Physics, 6:1-50.

Mardia, K. V. (1978). Some properties of classical multi-dimensional scaling. Communications in StatisticsTheory and Methods, A7:1233-1241.

Mardia, K. V., Kent, J. T., and Bibby, J. M. (1979). Multivariate Analysis. Academic Press, Orlando.

McCormick, G. P. and Tapia, R. A. (1972). The gradient projection method under mild differentiability conditions. SIAM Journal on Control, 10:93-98.

Meulman, J. J. (1992). The integration of multidimensional scaling and multivariate analysis with optimal transformations. Psychometrika, 57:539-565.

Parks, T. A. (1985). Reducible nonlinear programming problems. Technical Report 85-8, Department of Mathematical Sciences, Rice University, Houston, TX. Author's Ph.D. thesis.

Richardson, M. W. (1938). Multidimensional psychophysics. Psychological Bulletin, 35:659-660. Abstract.

Saito, T. (1978). The problem of the additive constant and eigenvalues in metric multidimensional scaling. Psychometrika, 43:193-201.

Schoenberg, I. J. (1935). Remarks to Maurice Fréchet's article "Sur la définition axiomatique d'une classe d'espaces distanciés vectoriellement applicable sur l'espace de Hilbert". Annals of Mathematics, 38:724732 .

Shepard, R. N. (1962). The analysis of proximities: Multidimensional scaling with an unknown distance function. II. Psychometrika, 27:219-246.

Takane, Y., Young, F. W., and de Leeuw, J. (1977). Nonmetric individual differences multidimensional scaling: An alternating least squares method with optimal scaling features. Psychometrika, 42:7-67.

Torgerson, W. S. (1952). Multidimensional scaling: I. Theory and method. Psychometrika, 17:401-419.

Trosset, M. W. (1990). A constrained optimization approach to nonmetric multidimensional scaling. In 1990 Proceedings of the Statistical Computing Section, pages 117-121. American Statistical Association.

Trosset, M. W. (1995). Distance matrix completion by numerical optimization. Technical Report 95-31, Department of Computational \& Applied Mathematics-MS 134, Rice University, Houston, TX 770051892.

Trosset, M. W. (1996). Numerical algorithms for multidimensional scaling. In Studies in Classification, Data Analysis, and Knowledge Organization. Gesellschaft für Klassifikation e.V., Springer-Verlag.

Trosset, M. W. (1997). Computing distances between convex sets and subsets of the positive semidefinite matrices. Technical Report 97-3, Department of Computational \& Applied Mathematics-MS 134, Rice University, Houston, TX 77005-1892. 
Trosset, M. W., Baggerly, K. A., and Pearl, K. (1996). Another look at the additive constant problem in multidimensional scaling. Technical Report 96-7, Department of Statistics-MS 138, Rice University, Houston, TX 77005-1892. Submitted for publication.

Wish, M. (1971). Individual differences in perceptions and preferences among nations. In King, C. W. and Tigert, D., editors, Attitude Research Reaches New Heights, pages 312-328. American Marketing Association, Chicago.

Wish, M., Deutsch, M., and Biener, L. (1972). Differences in perceived similarity of nations. In Romney, A. K., Shepard, R. N., and Nerlove, S., editors, Multidimensional Scaling: Theory and Applications in the Behavioral Sciences, volume 2, pages 289-313. Seminar Press, New York.

Young, F. W. and Levinsohn, J. R. (1974). SCALAR-1130 and SCALAR-360: two special purpose programs that perform Nonmetric Multidimensional Scaling. Journal of Marketing Research, 11:315-316.

Young, F. W. and Torgerson, W. S. (1967). TORSCA, a Fortran IV program for nonmetric multidimensional scaling. Behavioral Science, 12:498.

Young, G. and Householder, A. S. (1938). Discussion of a set of points in terms of their mutual distances. Psychometrika, 3:19-22. 
Figure 1

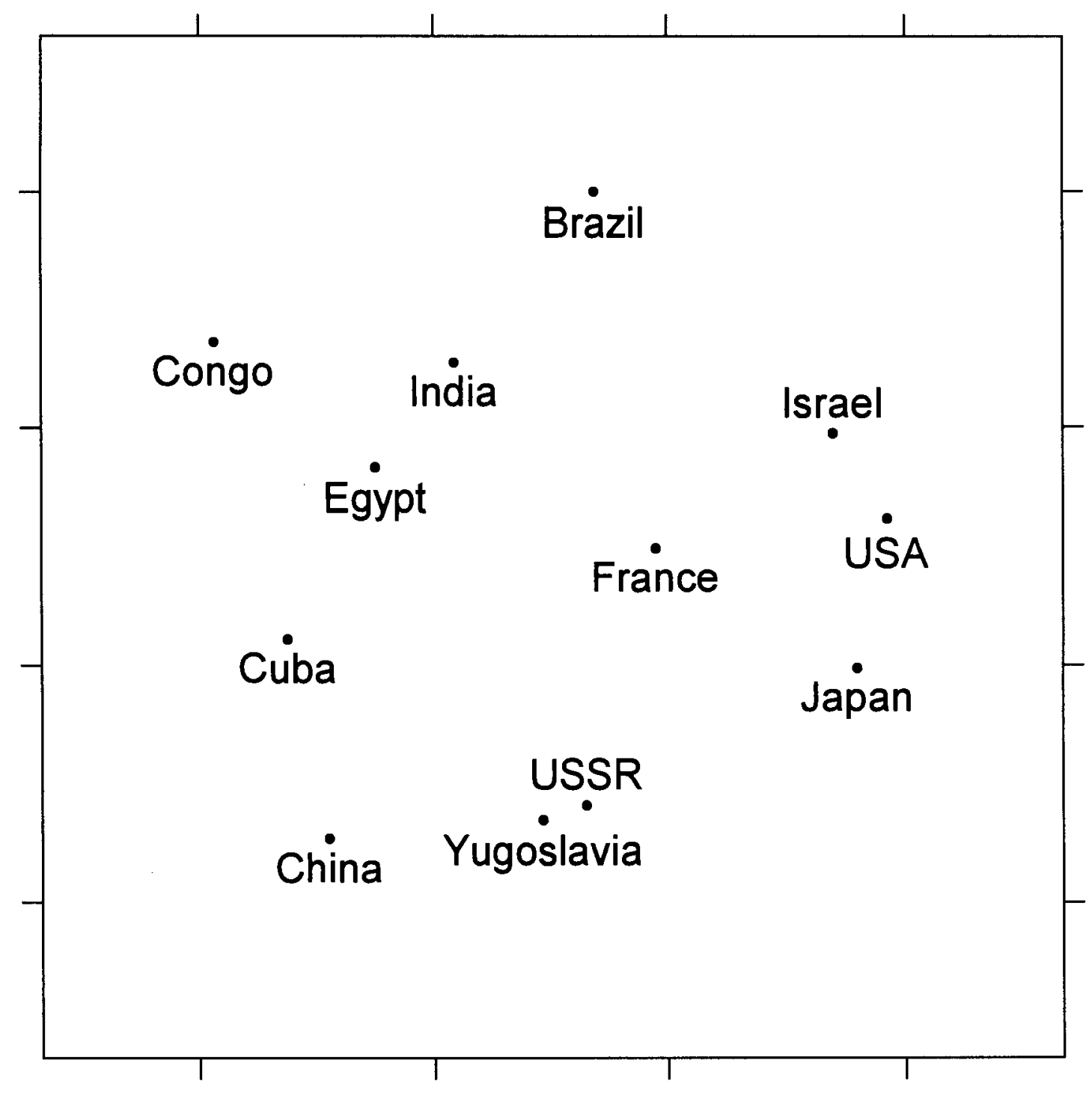

Nonmetric STRESS configuration for Wish's perceptions of nations data. 
Figure 2

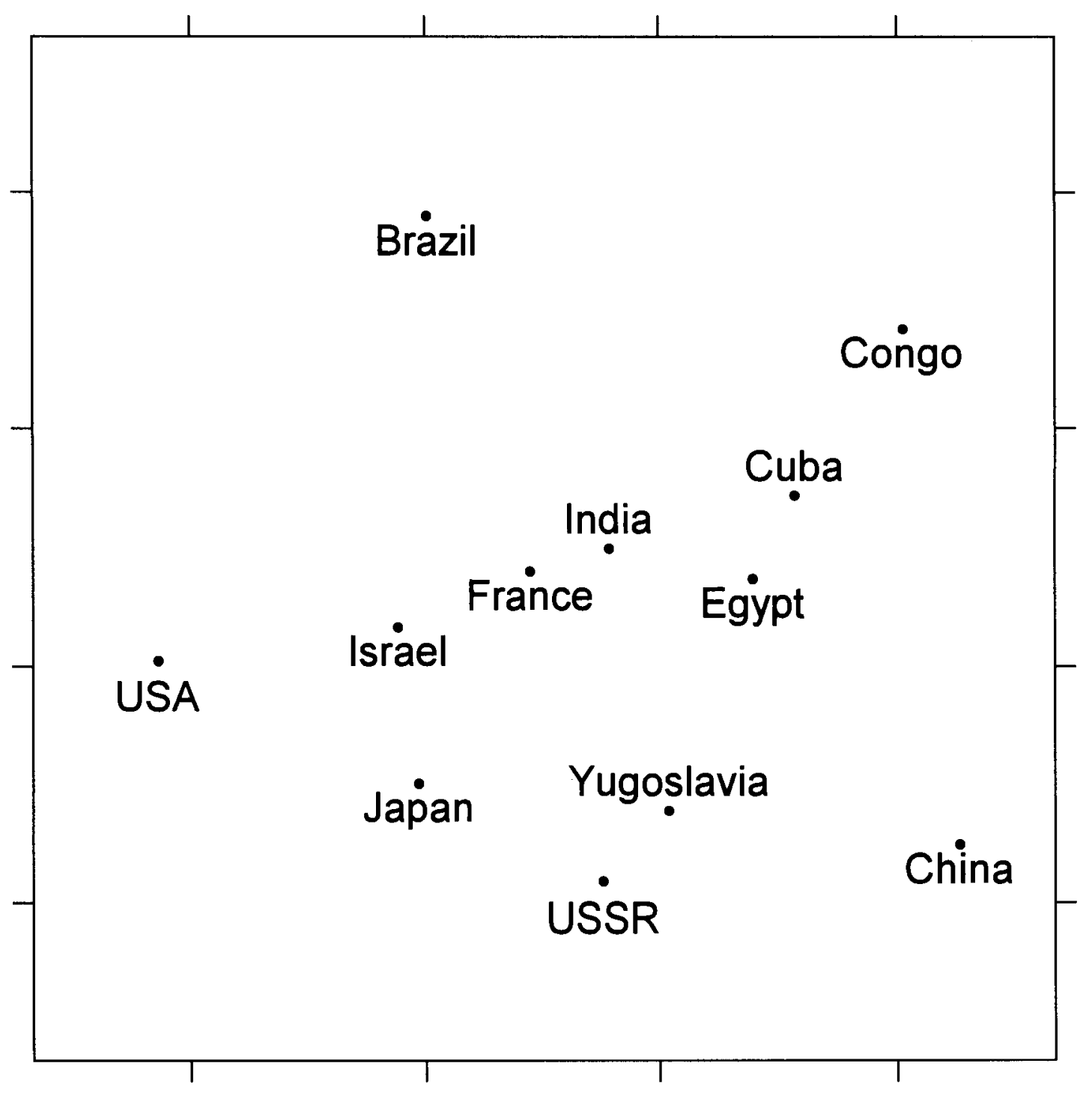

Nonmetric STRAIN configuration for Wish's perceptions of nations data. 
Figure 3

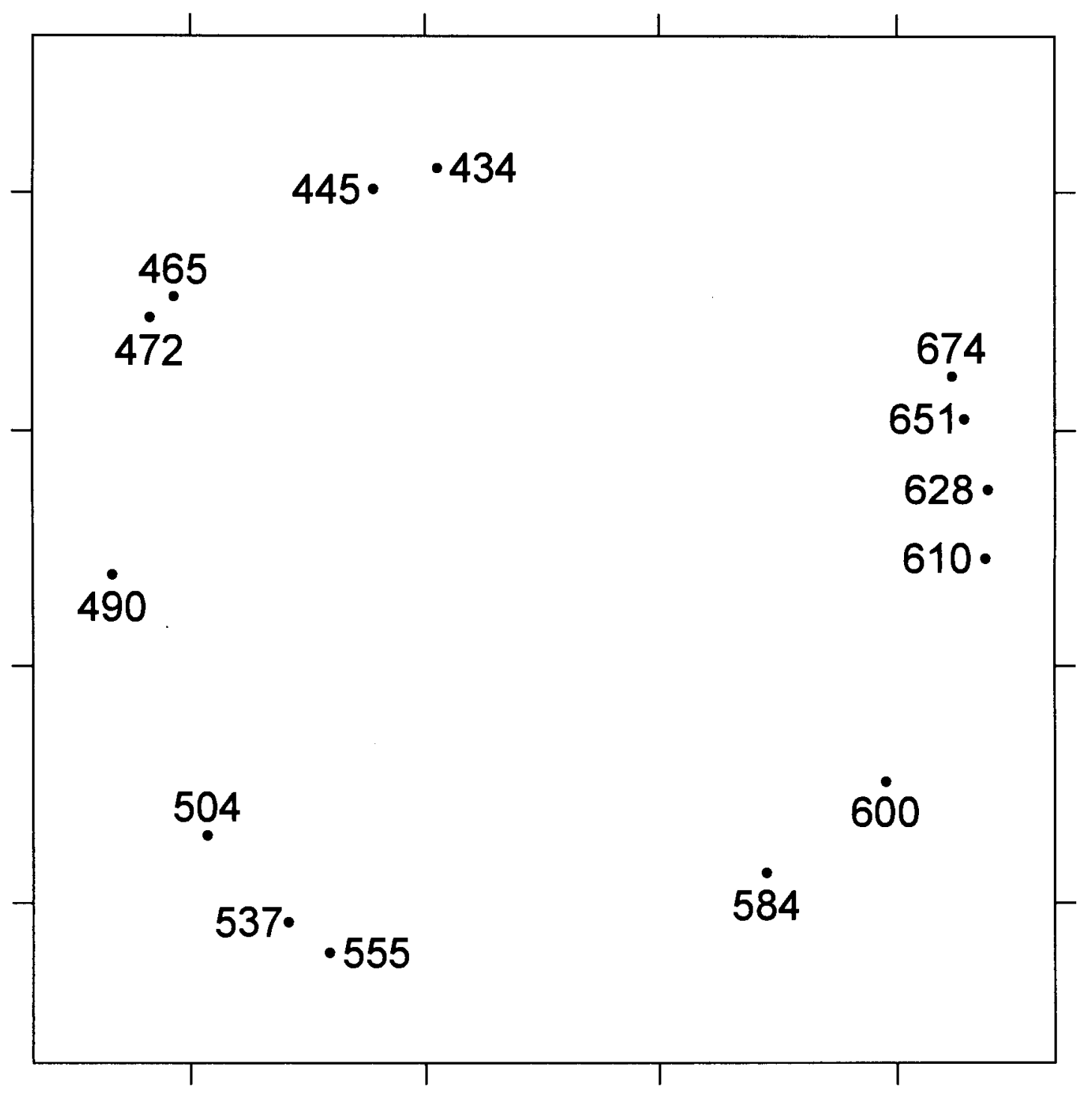

Nonmetric STRAIN configuration for Ekman's perceptions of colors data. 